\title{
Species temporal persistence promotes the stability of fruit-frugivore interactions across a 5-year multilayer network
}

\author{
José M. Costa ${ }^{1,2}$ (D) | Jaime A. Ramos ${ }^{2}$ (D) | Sérgio Timóteo ${ }^{1}$ (D) | Luís P. da Silva ${ }^{1,2}$ (D) | \\ Ricardo S. Ceia ${ }^{2}$ (D) | Ruben H. Heleno ${ }^{1}$
}

${ }^{1}$ Centre for Functional Ecology - Science for People \& the Planet, Department of Life Sciences, University of Coimbra, Coimbra, Portugal

${ }^{2}$ MARE-Marine and Environmental Sciences Centre, Department of Life Sciences, University of Coimbra, Coimbra, Portugal

Correspondence

Ruben H. Heleno

Email: rheleno@uc.pt

Present address

Luís P. da Silva, CIBIO-InBIO, Research

Centre in Biodiversity and Genetic

Resources, University of Porto, 4485-661,

Vairão, Portugal

Funding information

FCT/MEC, Grant/Award Number: UID/ BIA/04004/2019, SFRH/BD/96292/2013 and SFRH/BD/77746/2011; Marie Curie Actions, Grant/Award Number: FP7-PEOPL and E-2012-CIG-321794

Handling Editor: Ignasi Bartomeus

\section{Abstract}

1. Biological communities are intrinsically dynamic, with species and interactions changing over time. However, the temporal dynamics of species interaction networks is usually assessed using independent 'snapshot' networks, which may provide an incomplete representation of ecological processes. The use of temporal multilayer networks, where networks (i.e. layers) are formally interconnected via interlayer links, can circumvent such limitation, allowing for a more realistic characterization of community structure and better predictions regarding long-term processes that rely on species interactions, such as seed dispersal.

2. We used a 5-year bird-seed dispersal dataset to explore species and interaction turnover across years, network structure variability and the relationship between species topological roles on each year and their temporal persistence (i.e. species activity). We then implemented a temporal multilayer network approach to compute overall species versatility (centrality) and to reveal the modular structure of the 5-year multilayer network using changes in species relative abundances as proxies for the interlayer links between time-sequential networks.

3. Network topology remained relatively constant across years and interaction turnover was mostly due to true rewiring (new links) between species. Overall, birds, some of which migratory, were temporally more reliable than fleshy-fruited plants. Interestingly, species present across more years tended to be more important to the structure of each years' seed dispersal network, independently of their relative abundance. The multilayer analysis identified four modules of tightly interacting species, all of which spanning across the 5 years, and with a greater stability in the composition of bird species when compared to plants.

4. Synthesis. Bird and plant species that are present in more years in the seed dispersal network were also disproportionally important in each year, forming a core of temporally reliable interacting partners for transient species in the network.

KEYWORDS

community stability, ecological networks, frugivory, interaction persistence, multitrophic interactions, plant-animal mutualisms, seed dispersal, temporal dynamics 


\section{1 | INTRODUCTION}

The structure of ecological communities, reflected in the complex network of biotic interactions that connects co-occurring species, is intrinsically dynamic. Such dynamism may directly emerge from temporal changes on species composition (species turnover), animal feeding preferences (rewiring), relative species abundances and availability of suitable resources (phenological matching), such as flowers and fruits (Burkle \& Alarcón, 2011; Olesen et al., 2010; Trøjelsgaard \& Olesen, 2016). Although the dynamic nature of species interactions across time is widely recognized (Blonder, Wey, Dornhaus, James, \& Sih, 2012; Carnicer, Abrams, \& Jordano, 2008; Olesen et al., 2010; Trøjelsgaard \& Olesen, 2016), it is still rarely considered in ecological network studies. Some studies, mostly focusing on plant-pollinator interactions, started to explore the interannual variability on community structure (e.g. Chacoff, Resasco, \& Vázquez, 2018; Dupont, Padrón, Olesen, \& Petanidou, 2009; Petanidou, Kallimanis, Tzanopoulos, Sgardelis, \& Pantis, 2008; Rasmussen, Dupont, Mosbacher, Trøjelsgaard, \& Olesen, 2013); however, we still know relatively little about the yearly variation of plant-frugivore interactions whose studies have focused on shorter time scales (e.g. Carnicer, Jordano, \& Melián, 2009; GonzálezCastro, Yang, Nogales, \& Carlo, 2012; Ramos-Robles, Andresen, \& Díaz-Castelazo, 2016). Furthermore, although these studies provide important information about communities' structural changes across time, they still aggregate observed interactions into one or several formally disconnected time slices, likely providing an incomplete perception of true temporal dynamics (Blonder et al., 2012; Pilosof, Porter, Pascual, \& Kéfi, 2017). A multilayer network approach, where interdependencies between networks are explicitly incorporated in the analysis by quantifying the strength of interlayer links that represent biologically meaningful processes connecting species across multiple ordered networks, has a great potential to circumvent such limitations (Aleta \& Moreno, 2019; García-Callejas, Molowny-Horas, \& Araújo, 2018; Hutchinson et al., 2019; Pilosof et al., 2017).

Seed dispersal represents a key stage in the life history of most plant species, through which they can move away from mother plants and colonize new habitats (Traveset, Heleno, \& Nogales, 2014). Birds are critical seed dispersers across most terrestrial ecosystems, largely influencing habitat structure and long-term vegetation dynamics (Jordano, 2014; Wenny, Şekercioğlu, Cordeiro, Rogers, \& Kelly, 2016). In Southern Europe, a peak in seed dispersal occurs during late summer and early autumn when the production of fleshy fruits coincides with the occurrence of migratory birds that rely heavily on fruit consumption during this period (Carnicer et al., 2008; Herrera, 1984). Surprisingly, we still do not know how interannual fluctuations in species' relative abundances affect the persistence of plant-frugivore interactions, which limit the current understanding of long-term community structure and dynamics (Estes et al., 2018). For example: how plastic are fruit-frugivore interactions to changes on the availability of both interacting partners? Can we identify supra-annual modules of tight species interactions? Furthermore, the lack of such a long-term perspective is unanimously recognized as a major limitation to the understanding of biodiversity-ecosystem service relationships (Tilman, Isbell, \& Cowles, 2014).

Here, we explore the variability of bird-seed dispersal interactions across five consecutive years (2012-2016) in central Portugal at the peak of bird and fleshy fruit diversity. By combining standard monolayer networks across years and an overall temporal multilayer approach, we aim to (a) identify whether interannual interaction turnover is mostly determined by changes in species composition or by true interaction rewiring between temporally persistent species; (b) investigate the relationships between species topological roles in the community and their temporal persistence; (c) explore the interannual variability in monolayer network properties and (d) use a multilayer approach to identify groups of tightly interacting species (i.e. modules) and evaluate their temporal consistency.

\section{2 | MATERIALS AND METHODS}

\section{1 | Experimental approach}

For five consecutive years, we reconstructed the network of interactions between birds and fleshy-fruited plants on a secondary native forest in Central Portugal $\left(40^{\circ} 19^{\prime} \mathrm{N} ; 8^{\circ} 24^{\prime} \mathrm{W}\right)$. The site is under a Mediterranean climate and is dominated by Quercus faginea, Arbutus unedo and Pinus pinaster with a dense and diverse understorey dominated by native fleshy-fruited shrubs, such as Pistacia lentiscus, Crataegus monogyna, Rhamnus alaternus and Viburnum tinus.

Interaction networks were assembled by identifying entire seeds on the droppings of mist-netted birds captured under two complementary designs: (a) sampling all days with favourable weather conditions in September 2012 to 2016 (encompassing to the peak of seed dispersal and passage of migratory bird species at the study site) and (b) sampling fortnightly from January to December 2013 to evaluate the intra-annual richness of pairwise seed-bird interactions (hereafter, links). The intensive sampling in September was performed to obtain highly resolved data characterizing bird-seed interactions during that period, which allowed a robust assessment of interannual community dynamics (Blonder et al., 2012). Additionally, it also minimized the introduction of forbidden links due to mismatches between bird migration and fruit phenology, which were likely to occur if we sampled throughout the entire fruiting season, ending up with an aggregated network. Each day, birds were captured with mist nets (total length $=102 \mathrm{~m}$ ) operated during the first $5 \mathrm{hr}$ after dawn and placed in individual cotton bags until they defecate or up to $30 \mathrm{~min}$. All droppings retrieved from the bags were air-dried and undamaged seeds were later extracted, counted and identified under a dissecting microscope with the aid of a seed reference collection. Interaction frequency was quantified as the number of droppings of bird species i containing undamaged seeds of plant species $j$. The overall effort resulted in 515 sampling hours distributed along 25, 17, 20, 21 and 20 days in September 2012 to 2016 , respectively, and $120 \mathrm{hr}$ in 24 additional days across the entire year of 2013. Sampling completeness was 
estimated for each year as the proportion of plant and bird species observed relative to those estimated by the Chao 2 richness estimator (Chao, 1987) implemented in software EstimateS 9.1 (Colwell, 2013). All bird species dispersing seeds were classified as migratory (occurring only seasonally in the study area), partially migratory (migratory with some resident individuals) and resident species (see Costa et al., 2014; Table S1). Additionally, fleshy-fruit availability was estimated by counting all ripe standing fruits along three linear transects (each: $25 \mathrm{~m} \times 2 \mathrm{~m}$ ) running parallel to the mist nets and resampled each year in early, mid- and late September. These transects characterize fruit availability in the focal patch of continuous forest. In the few cases where seed species were found in the bird droppings but not in the fruit-availability transects, these were attributed the lowest availability score (1 fruit/transect) under the rationale that those fruits need to be available in order to be consumed but are rare in the forest and were probably consumed in the surrounding habitats (Table S2).

\subsection{Species and interaction turnover}

The interannual turnover of bird and plant species was assessed with the Whittaker beta diversity index $\left(\beta_{\mathrm{W}}\right)$ adapted by Koleff, Gaston, and Lennon (2003), which varies between 0 (similar) and 1 (completely dissimilar species composition). The turnover of links was evaluated with package BETALINK (Poisot, 2016) in R ( $R$ Core Team, 2017), following the approach outlined in Poisot, Canard, Mouillot, Mouquet, and Gravel (2012), which allows the partition of link turnover $\left(\beta_{\mathrm{WN}}\right)$ between networks into two driving mechanisms: those attributed exclusively to species turnover $\left(\beta_{\mathrm{ST}}\right)$ and those attributed to the true rewiring of interactions between temporally persistent species $\left(\beta_{\mathrm{OS}}\right)$.

\section{3 | Relationship between species temporal persistence and their topological roles}

To characterize the regularity of species across the 5 years, we propose a new species-level index, which we coined 'species activity', that quantifies the number of networks in which each species interacts (i.e. with 'activity' of each species). This descriptor is a direct extension of the concept of 'node activity' used in physics to reflect the number of layers (i.e. networks) where the nodes of multiplex networks are 'active' (Nicosia \& Latora, 2015). We then evaluated how species activity is related to species' topological importance in each year, by computing three monolayer species-level descriptors for each years' network: (a) degree, that is, the number of interacting partners; (b) species strength, an estimation of the cumulative importance of species $i$ for all species on the other trophic level (Bascompte, Jordano, \& Olesen, 2006) and (c) specialization d', quantifying species selectivity in relation to resource availability determined by the observed interaction matrix marginal totals (Blüthgen, Menzel, \& Blüthgen, 2006).
In addition, we computed species' multilayer versatility, a measure of centrality, in order to assess the importance of each species to the cohesiveness of the overall (supra-annual) network by connecting different parts of the temporal multilayer network, both within and between layers (De Domenico, Solé-Ribalta, Omodei, Gómez, \& Arenas, 2015; Timóteo, Correia, Rodríguez-Echeverría, Freitas, \& Heleno, 2018). Species versatility was computed using the PageRank algorithm (Brin \& Page, 2012), which performs a random walk through existing intralayer links with a probability $p$ and a 'teleportation' with a probability $1-p$ to any node in the multilayer network (De Domenico, Solé-Ribalta, et al., 2015), in program muxViz (De Domenico, Porter, \& Arenas, 2015). We defined our multilayer network as ordinal and choosing the option 'edge-colored network' to allow only teleportation from layer $(t)$ to layer $(t+1)$, thus preserving the temporal nature of the seed dispersal multilayer network. Since species versatility assumes unipartite networks as inputs, its calculation was done separately for bird and plant species based on unipartite projections of the original networks using the Newman's method (Newman, 2001) adapted for weighted networks (Opsahl, 2013) with the R package TNET (Opsahl, 2009).

In order to assess if plants were dispersed proportionally to their abundance on each year, we calculated the Kendall's tau rank correlation coefficient between the abundance of fleshy fruits in transects and their respective interaction frequency for each species, using the R function 'cor.test()'. The relation between species activity and species degree and strength was assessed with GLMM with a Poisson and Gamma error distribution respectively. In order to control for the effect of variable network sizes (which may directly influence species degree in each year), the logarithm of the number of potentially interacting species (i.e. number of plant species for bird degree and the reverse) was included as an offset variable in the Poisson GLMM. The relationship between species activity and species specialization $d^{\prime}$ was modelled with a linear mixed model (LMM). The relationship between species versatility and species activity was assessed with a GLM with a Gamma error distribution. The GLMs were fitted with the ' $g \operatorname{lm}()$ ' function in $\mathrm{R}$, the statistical significance $(p<0.05)$ of the estimates was assessed from likelihood ratio tests with the 'ANOVA()' function, and $95 \%$ profile likelihood confidence intervals from the 'confint()' function. The significance of the fixed effects was assessed with parametric bootstrapped $p$-values (1,000 simulations) obtained with the R package AFEx (Singmann, Bolker, Westfall, Aust, \& Ben-Shachar, 2020), and 95\% parametric bootstrapped confidence intervals (1,000 simulations) from the 'confint.merMod()' function in LME4 package (Bates, Maechler, Bolker, \& Walker, 2015). To assess if migratory bird species were topologically more important to the seed dispersal network than residents, we used GLMs with bird migratory strategy (partially migratory, migratory or resident species) as dependent variable and $d^{\prime}$ (LMM), degree (Poisson GLMM with the log number of plant species as offset) and strength (Gamma GLMM) as independent variables. The overall significance $(p<0.05)$ of bird migration strategy was assessed with parametric bootstrapped $p$-values (1,000 simulations) as described above. All mixed models included year as a random factor. 


\section{4 | Interannual community structure}

Changes in the emergent structure of each years' monolayer seed dispersal network were evaluated by calculating three network-level descriptors: (a) connectance, the proportion of observed/possible links (Jordano, 1987); (b) network specialization $\mathrm{H}_{2}{ }^{\prime}$, measuring the community-level selectiveness of the observed interactions as a departure from a random (i.e. abundance-based) association pattern (Blüthgen et al., 2006) and (c) weighted-interaction nestedness (WIN; Galeano, Pastor, \& Iriondo, 2009), quantifying how interactions are hierarchically organized (i.e. nested) around a core of the most generalist species (Bascompte, Jordano, Melián, \& Olesen, 2003). These descriptors were selected because they are among the most widely used and because they have been shown to carry important information regarding network stability (Thébault \& Fontaine, 2010); although the value of nestedness has been challenged, it is still commonly used (Payrató-Borràs, Hernández, \& Moreno, 2019). The significance of each descriptor was assessed by comparing it with those obtained for 1,000 randomized networks generated by a null model based on the Patefield's algorithm (Patefield, 1981), which randomly reshuffles the interactions across the matrix while constraining marginal totals. Following a normal distribution, each descriptor was considered significantly different from a random expectation if the respective $z$-score was lower than -1.96 or higher than 1.96 standard deviations from the mean (Trøjelsgaard, Jordano, Carstensen, \& Olesen, 2015). These network descriptors and respective null models were computed with the R package BIPARTITE (Dormann, Fründ, Blüthgen, \& Gruber, 2009; Dormann, Gruber, \& Fründ, 2008).

\section{5 | Multilayer modularity}

The identification of modules of strongly interacting species has gained increasing attention in ecology due to their importance for ecological and evolutive processes (Nogales et al., 2015; Olesen, Bascompte, Dupont, \& Jordano, 2007). Here, we identified such modules based on an explicit multilayer network, thus allowing modules to span and link multiple layers (Timóteo et al., 2018). To this end, species occurring in sequential networks are assumed to be connected by interlayer links, here quantified as the change on relative abundance of species' $i$ between consecutive years (i.e. abundance $i(t+1)$ /abundance $i(t)$; see also Pilosof et al., 2017). Plant abundance was measured as the mean number of fruits per transect and bird abundance as the mean number of birds captured per day. While the best way to quantify interlayer link strength is still an open question (see Section 4), changes in species relative abundances seems a good indicator of population processes (e.g. survival and reproduction) linking consecutive years (Pilosof et al., 2017). When a species $i$ was not present in any of the two consecutive networks the interlayer link was set to zero. Modularity was maximized in 1,000 runs with a generalized Louvain algorithm (Blondel, Jean-Loup, Renaud, \& Etienne, 2008) implemented in MATLAB (The MathWorks, Inc.) using code provided in Jutla Jeub and Mucha (2014) and modified by Pilosof et al. (2017) to account for the bipartite nature of the multilayer network (Timóteo et al., 2018). The significance of observed modularity was assessed against the modularity distribution from 1,000 randomized networks, created with the Patefield algorithm in the R package vegAn ('r2dtable' option; Oksanen et al., 2015).

\section{3 | RESULTS}

Throughout 2013 (fortnight sampling) we captured 671 birds from 30 species, whose 202 droppings contained 537 undamaged seeds from 16 plant species. September was the month with a greater diversity of links between fleshy-fruited plants and birds (Figure S1).

Overall, the daily sampling throughout September 2012 to 2016 resulted in the capture of 1,620 birds (30 species), of which 454 (12 species) dispersed 2,133 undamaged seeds from 17 plant species (Figure 1). Estimated sampling completeness was very high for both plants and birds, with an annual mean of 93\% (Min. =90\%; Max. =98\%) and $92 \%$ (Min. $=89 \%$; Max. $=100 \%$ ) of species detected respectively.

\section{1 | Species and interaction turnover}

Five plant species (Ficus carica, R. alaternus, Rubus ulmifolius, Smilax aspera and Vitis vinifera) and six bird species (Erithacus rubecula, Ficedula hypoleuca, Sylvia atricapilla, S. borin, S. melanocephala and Turdus merula) were detected in all the 5 years of the study (Figures 1 and 2). These species accounted for $29 \%$ of the fruit production and $50 \%$ of the individual birds captured in September across the 5 years. Mean species turnover between consecutive years $\left(\beta_{\mathrm{W}}\right)$ was higher for plants than for birds (mean $\pm S D: 0.31 \pm 0.12$ and $0.16 \pm 0.07$ respectively). Nine out of the 75 links detected (12\%) were observed in all years, accounting on average for $49 \%$ (range $=30 \%-63 \%$ ) of the links detected in each year. The turnover of interactions was greater than that of plant and animal species $\left(\beta_{\mathrm{WN}}=0.53 \pm 0.10\right)$ and mostly driven by true interaction rewiring, that is, the detection of new links between species already co-occurring on previous years $\left(\beta_{\mathrm{OS}}=0.37 \pm 0.09\right)$, with a lower contribution of species turnover $\left(\beta_{\mathrm{ST}}=0.16 \pm 0.07\right)$.

\section{2 | Relationship between species temporal persistence and their topological roles}

There was no significant correlation between fruit abundance and the frequency of interactions in any year $\left(\tau_{2012}=0.12, p=0.66\right.$; $\tau_{2013}=0.60, p=0.11 ; \tau_{2014}=0.53, p=0.05 ; \tau_{2015}=0.44, p=0.13$; $\left.\tau_{2016}=-0.32, p=0.45\right)$. Species activity of both plants and birds was positively associated with their mean annual degree, mean annual species strength and multilayer versatility (Table 1; Figure 2). In contrast, plant and bird specialization $d$ ' was not associated with species activity (Table 1; Figure 2). No significant differences 


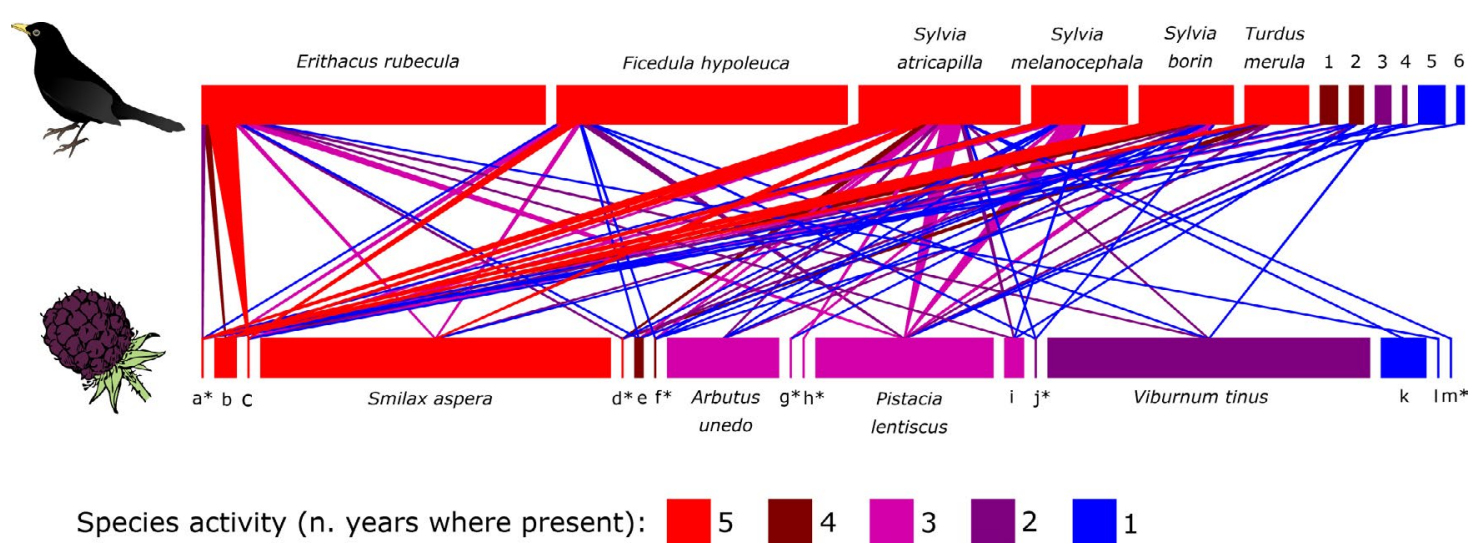

FIGURE 1 Overall seed-dispersal network recorded during the peak of the fruiting season (September) across five consecutive years on a secondary native forest in central Portugal. Species are ordered from highest to lowest species activity, that is, number of years where present. The width of the boxes representing plant and bird species is proportional to the number of fruits counted along linear transects and to the number of birds captured with mist-nets, respectively. Asterisks $\left(^{*}\right)$ represent seed species recovered from bird droppings but not detected in the transects. 1 - Cyanistes caeruleus, 2 - Sylvia communis, 3 - Muscicapa striata, 4 - Sylvia undata, 5 - Chloris chloris, 6 - Dendrocopos major; Plants: a - Ficus carica, b - Rhamnus alaternus, c - Rubus ulmifolius, d - Vitis vinifera, e - Phillyrea latifolia, f - Solanum nigrum, g - Daphne gnidium, h - Lonicera periclymenum, i - Rubia peregrina, j - Phytolacca americana, k - Crataegus monogyna, I - Olea europaea, $\mathrm{m}$ - Phillyrea angustifolia

FIGURE 2 Topological descriptors of species roles on avian seed-dispersal networks compiled over 5 years. The top panel corresponds to species roles on a temporal multilayer network, while the monolayer panels reflect average species roles across the yearly networks where each species occurs. Error bars represent the standard error for each descriptor across the 5 years. Bars without error bars correspond to species with no interannual variation to a given descriptor. Species are ordered according to their overall relative abundance (Bird species: number of birds captured; Seed species: abundance of fruits in the transects)

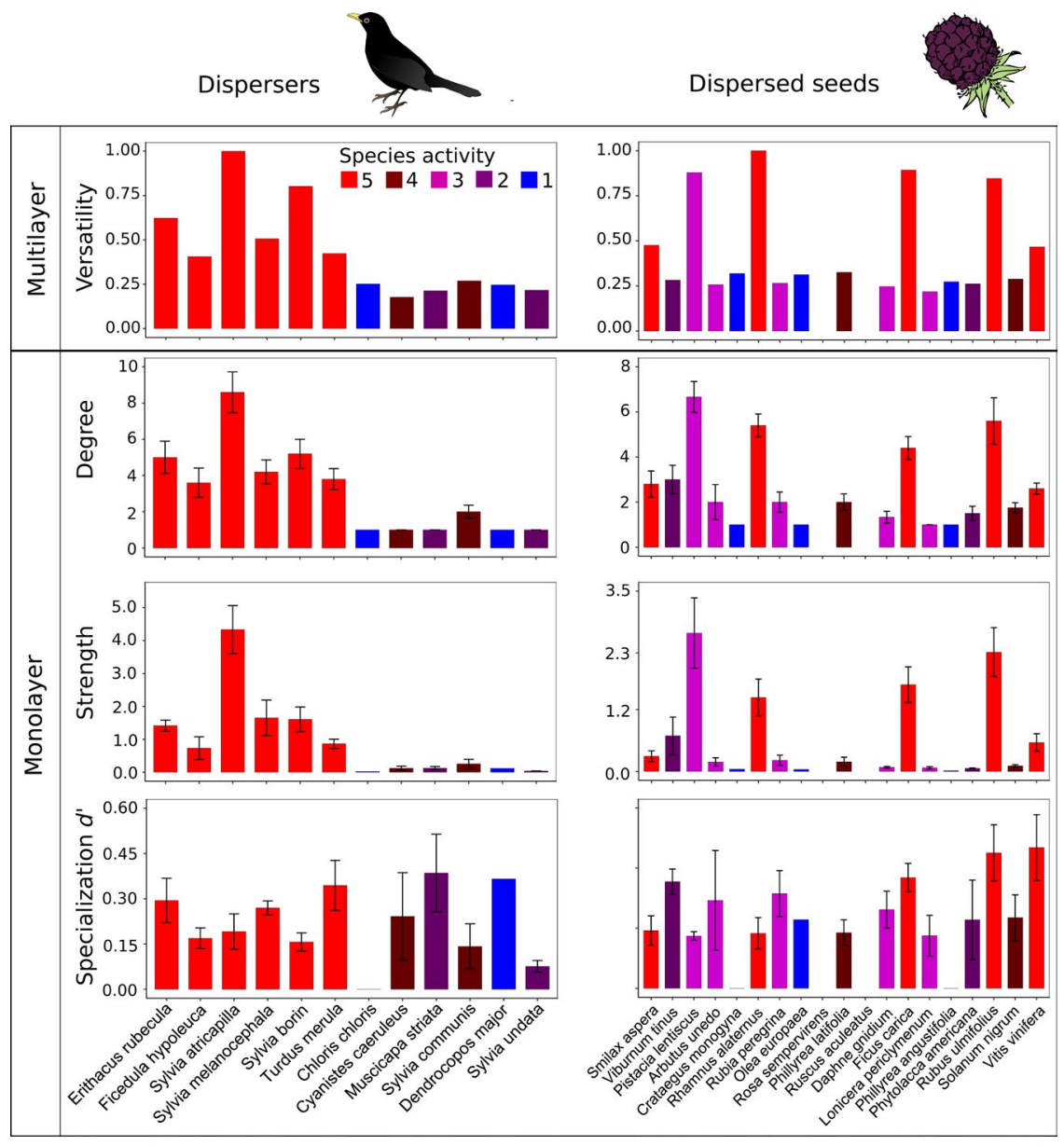

were detected in specialization $d^{\prime}$ among bird migratory strategies (Table 2; Figure S2). Partially migratory bird species dispersed more plant species and had a higher species strength than migratory and resident species (Table 2; Figure S2).

\section{3 | Interannual community structure}

Overall, the topology of the annual networks was relatively constant throughout the study (Figure S3). All annual networks were 
TAB LE 1 Results of the statistical analysis on the relationship between species' network roles and temporal persistence (species activity). 95\% Cl: 95\% likelihood profile and 95\% parametric bootstrapped confidence intervals (GLMs and (G)LMMs respectively). Statistically significant relationships $(p<0.05)$ are marked with an asterisk

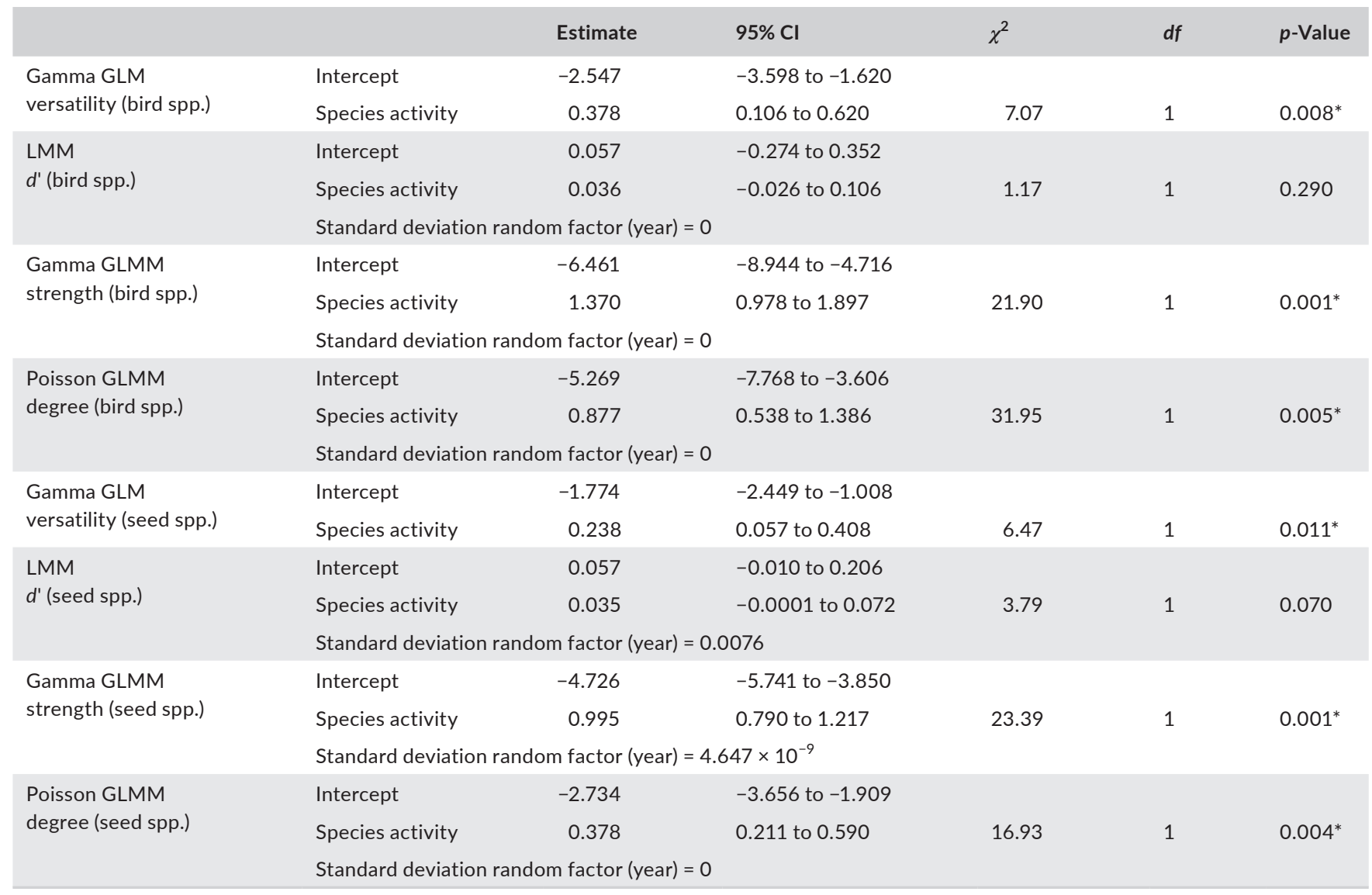

\begin{tabular}{llrl}
\hline Model & Pairwise comparison & Estimate & \multicolumn{1}{l}{$95 \% \mathrm{Cl}$} \\
\hline LMM $\left(d^{\prime}\right)$ & SDM-R & -0.010 & -0.165 to 0.145 \\
SD random = & LDM-R & -0.064 & -0.202 to 0.067 \\
& LDM-SDM & -0.058 & -0.216 to 0.101 \\
& Bird species. Migratory strategy: $\chi^{2}=1.58, d f=2, p=0.50$ \\
\hline Gamma GLMM (strength) & SDM-R & 1.361 & 0.359 to $2.364^{*}$ \\
SD random = 0 & LDM-R & 0.094 & -0.779 to 0.967 \\
& LDM-SDM & -1.267 & -2.291 to $-0.242^{*}$ \\
\hline Poisson GLMM (degree) & Bird species. Migratory strategy: $\chi^{2}=12.43, d f=2, p=0.003^{*}$ \\
\hline SD random = 0 & SDM-R & 0.961 & 0.519 to $1.403^{*}$ \\
& LDM-R & 0.222 & -0.243 to 0.686 \\
& LDM-SDM & -0.740 & -1.167 to $-0.313^{*}$ \\
\hline & Bird species. migratory strategy: $\chi^{2}=29.88, d f=2, p=0.004^{*}$ \\
\hline
\end{tabular}

TABLE 2 Results of the models fitted to compare species' network roles between bird species migratory strategies. 'SDM', short-distance migratory; 'LDM', long-distance migratory; ' $R$ ', resident; '95\% Cl', 95\% simultaneously confidence intervals; 'SD random', standard deviation of the random factor (i.e. year). Statistical significance is marked with an asterisk significantly less connected $\left(z_{2012}=-5.12 ; z_{2013}=-2.97 ; z_{2014}=-2.15\right.$; $\left.z_{2015}=-3.31 ; z_{2016}=-4.51\right)$ and more specialized $\left(z_{2012}=9.67\right.$; $z_{2013}=3.90 ; z_{2014}=3.16 ; z_{2015}=5.86 ; z_{2016}=8.14$ ) than predicted by the null models. The networks also tended to be significantly nested, which happened in all years except in 2014, when observed nestedness was indistinguishable from a random interaction pattern $\left(z_{2012}=4.22 ; z_{2013}=3.43 ; z_{2014}=0.93 ; z_{2015}=3.91 ; z_{2016}=5.41\right)$.

\section{4 | Multilayer modularity}

The overall multilayer network was significantly more modular than expected by chance (mean $Q_{\text {obs }}=0.50$ [range 1,000 runs $=0.48$ 0.50 ], mean $Q_{\text {null }}=0.43$ [range 1,000 runs $=0.41-0.45$ ]), and formed by four interaction modules (Figure 3 ), all of which spanned across the 5 years of the study. Most bird species that dispersed seeds in 


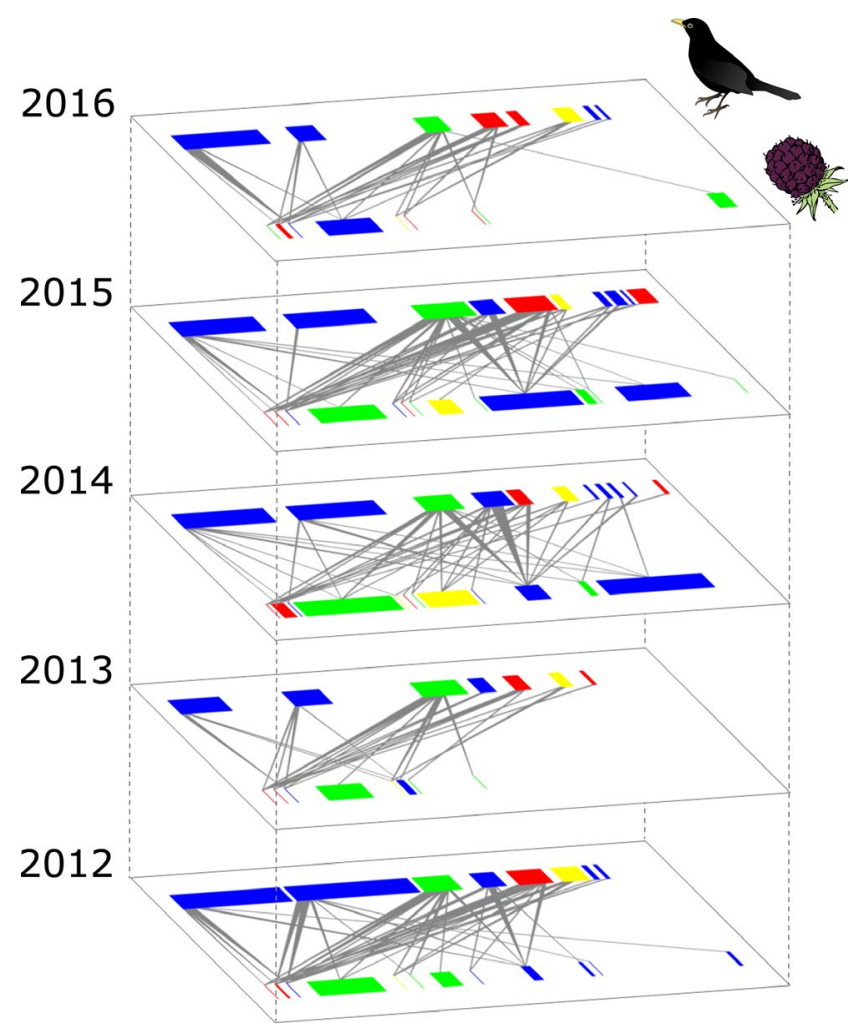

FIGURE 3 Interannual module affiliation of species across a 5-year temporal multilayer seed dispersal network. Colours represent different interaction modules (see Section 2). All networks are represented on the same scale and species are ordered as in Figure 1. The width of the boxes representing plant and bird species is proportional to the number of fruits counted along linear transects and to the number of birds captured with mist nets respectively

at least 2 years were consistently allocated into the same module across all years (eight out of 10 species, 80\%). In turn, plants had a lower temporal constancy regarding their module affiliation with only six out of the 14 plant species (43\%) remaining in the same module across all years.

\section{4 | DISCUSSION}

Here, we show that the diversity of seed dispersal interactions between birds and fleshy-fruited plants in Portugal is particularly high in September, when a peak in fruit production coincides with the passage of most migratory bird species. We compiled seed dispersal interactions during this activity peak for five consecutive years to reconstruct a temporal multilayer network, simultaneously quantifying intra- and interlayer (i.e. year) link strength. We found that the species present in more years (here said to have a greater species activity) also tend to be the more important species in each year. The structure of the seed dispersal network was very stable through time and included four well-defined interaction modules spanning across all years of the study. Altogether, our results suggest that the temporally most reliable species, which are not necessarily the most abundant, tend to occupy central roles in the seed dispersal network across multiple temporal scales increasing the reliability of the seed dispersal service.

We introduce species activity as a species-level descriptor that quantifies the role of each species as connectors of layers in a multilayer network, although it can also be used outside an explicit multilayer context (i.e. without interlayer links). Similar indices have been implicitly used to explore the persistence of species across years in flower-visitor networks (Olesen, Stefanescu, \& Traveset, 2011; Petanidou et al., 2008), months in bird-fruit networks (Ramos-Robles et al., 2016; Yang, Albert, \& Carlo, 2013), and across multiple habitats in seed dispersal networks (Timóteo et al., 2018). However, until now this index did not receive a common nomenclature or a standardized definition, rendering its use particularly difficult and resistant to generalization. Species activity can also be used in the context of spatial networks (number of sites/habitats/plots where present) or multiple functional layers (number of trophic levels or interaction types where present), thus being a highly versatile and intuitive metric to explore networks across several scales.

\subsection{Species and interaction turnover}

The low species turnover and the high contribution of true rewiring to interannual network dissimilarity suggest that most of the links tend to occur between temporally persistent species. Although only $12 \%$ of the links were detected on the 5 years, these links were disproportionally frequent each year (see also Chacoff et al., 2018, for a similar finding in a plant-pollinator network), suggesting that birds tend to interact disproportionally with temporally reliable fruiting plants independently of their abundance. It is possible that this lack of significant correlation between fruit abundance and interaction frequencies might have been ameliorated if we increased the number of fruit-availability transects. However, our transects covered a relatively large area (150 square-metres) on a homogeneous forest patch and therefore seem to provide a good proxy for real fruit availability in the forest. Additionally, the dispersed plant species that were not found inside the forest were very likely consumed in marginal habitats, mostly in agricultural land some $200 \mathrm{~m}$ away from the bird-capture site. The temporal persistence of species was more evident for birds than for plants. These results contrast with previous findings from plant-pollinator networks, where turnover tends to be greater for animals than for plants (Cirtwill, Roslin, Rasmussen, Olesen, \& Stouffer, 2018; Dupont et al., 2009; Petanidou et al., 2008), but it may be expected in bird-seed dispersal due to the high interannual variability in fruit production (Herrera, 1998). Similarly, variations in the timing of fruit ripening will also contribute to increase plant species turnover. The lower turnover of bird species is explained by the predictable occurrence of most species in the study area during September. 
Here, the role of rewiring is better interpreted as the contribution of the reshuffling of interactions between temporally persistent species (e.g. CaraDonna et al., 2017). However, interaction rewiring sensu lato may also occur as a consequence of species turnover, such as when one species interacts with a recently arrived species. In this study, the contribution of species turnover to network dissimilarity seems to have been partially driven by the irregular presence of $P$. lentiscus, an important fruit resource available only in 3 years (out of the 5 years) of the study. In the other 2 years, $P$. lentiscus fruits have likely matured only after September, and migratory species had to turn to other available fruits during their September migration through the area. This finding is consistent with the results of Rumeu, Álvarez-Villanueva, Arroyo, and González-Varo (2019) where the presence of highly nutritious $P$. lentiscus fruits reduced the consumption of other, less rewarding fruit species.

\section{2 | Relationship between species temporal persistence and their topological roles}

The bird and plant species with higher species activity were consistently the most important species in the annual networks because of their high number of interacting partners and interaction frequency. Consequently, these species tend to occupy a central position across the overall temporal multilayer network, assuming a disproportional role to the cohesion of its structure. These birds include mostly migratory and partially migratory species, notably S. borin and S. atricapilla, respectively, but also some resident birds that increase substantially their frugivorous behaviour when ripe fleshy fruits become available (Bairlein, 2002; Carnicer et al., 2008). Conversely, bird species with lower species activity and lower importance to the seed dispersal network essentially include granivorous and insectivorous species that only occasionally disperse unharmed seeds from a few plant species (Costa et al., 2018). The finding that both migratory and partially migratory birds tend to be more important as seed dispersers of fleshy-fruited plants agrees with previous findings in similar habitats (Herrera, 1998). As for plants, although the most important species in each year also tend to be the species with higher species activity, the exception to this is P. lentiscus, which is very important when present, but it is not a temporally reliable resource, at least for migratory birds. In contrast to the other species-level descriptors, we did not find differences in species specialization $d^{\prime}$ among neither bird nor plant species with different species activity. This descriptor is a measure of reciprocal interaction exclusiveness (Blüthgen et al., 2006). In our study, species with low degree tended to interact with species having a high interaction degree, irrespective of species activity, originating similar levels of $d^{\prime}$ for all species (Figure 2). Overall, these results indicate that bird species with high species activity tend to be more reliable seed dispersers. In turn, plants with high species activity provide high-quality resources for migrant birds that rely on fruits to increase their body fat reserves for the migratory flights (Bairlein, 2002).

\section{3 | Interannual community structure}

We found a highly stable interannual network structure, with the only exception of 2014, when the network was not significantly nested. While the stability of seed dispersal network structure across seasons has already been noted (Plein et al., 2013), our study suggests that such stability can also be extended to interannual network structure, following the patterns observed in pollination networks (Dupont et al., 2009; Petanidou et al., 2008). Our 5-year study also shows that, as expected on any ecological process, not all years are exactly equal and that extrapolations based on temporally restricted sampling (such as nestedness in 2014) may lead to a biased characterization of network structure (Estes et al., 2018).

\subsection{Multilayer modularity}

The identification of tight interaction modules within the relatively loose interaction networks has been one of the most insightful advances in community ecology of the last decades (Olesen et al., 2007; Schleuning et al., 2014). Such modules are constrained by species' morphological and behavioural traits influencing feeding preferences and habitat use (Donatti et al., 2011; Schleuning et al., 2014) and have important ecological and evolutionary implications (Nogales et al., 2015). In our study, module composition was relatively stable across years, with most species, particularly birds, maintaining their module affiliation through time, suggesting that bird species might have consistently distinct fruit preferences that are independent of fruit abundance. For example, R. ulmifolius, which was one of the most dispersed plant species, was rarely dispersed by $S$. atricapilla. In contrast, this bird species was one of the main seed dispersers of $F$. carica, which was less frequently dispersed by most other bird species (Figure 1). The largest module included bird species with a moderate dependence on fruits (mostly insectivorous and omnivorous) that disperse seeds from only a few plant species (mostly R. ulmifolius and P. lentiscus). A second group is dominated by $T$. merula, a medium-sized passerine that tends to disperse seeds from relatively larger sized fruits (e.g. V. vinifera). Finally, two modules were dominated by two highly frugivorous species: S. atricapilla and S. borin respectively. Although there is a considerable overlap in the identity of dispersed seeds by these two species, their separation in two modules suggests that they have slightly different diet preferences. Contrasting to birds, most plant species occurred in at least two modules throughout the study period (Figure 3). This suggests that there are interannual fluctuations in the frequency of interactions that may result from changes in relative 
fruit availability, for example due to variations in plant phenology constraining seed dispersers' preferences (Carlo \& Morales, 2008; Donoso, García, Martínez, Tylianakis, \& Stouffer, 2017; Rumeu et al., 2019).

By using a multilayer approach, we were able to identify tight interaction modules spanning across several years, bringing us closer to understand the long-term dynamics of natural communities. However, observed multilayer modularity might be affected by the weight of the interlayer links (Timóteo et al., 2018). Although it is analytically possible to assume uniform interlayer links for all species (Timóteo et al., 2018), this is likely unrealistic, and a quantification of interlayer link strength for each species is thus recommended (Hutchinson et al., 2019). In our study, we used interannual changes in species relative abundances as a proxy for species survival, allowing to combine information on species' population dynamics to community structure. However, the best metric to characterize interlayer link strength is still open to debate and it can be highly specific to each particular system (Hutchinson et al., 2019). Although multilayer networks have a high potential to increase the realism of network representations and analysis, they are not a key to every door. Therefore, we envisage that monolayer network approaches will continue to be useful to answer different questions, particularly when combined with multilayer approaches operating on complementarity scales.

\section{5 | CONCLUSIONS}

Understanding how biotic interactions span across multiple temporal scales has a great potential to improve the realism of our network representations and bring us closer to a predictive community ecology. Here, we combined a classic monolayer with a temporal multilayer approach to understand the interannual and supra-annual dynamics of bird-seed dispersal networks. Despite a high interannual interaction turnover, network structure and composition were remarkably stable across years, including migratory bird species, and consistently dominated by a few very abundant links. These persistent species (here defined as having high species activity) are also quantitatively the most important in each year in terms of the number of interacting partners and species strength, independently of fruit availability. Therefore, our study suggests that fruit-frugivore interactions are structured around a core of temporally reliable species, with which transient species tend to interact. The assessment of multilayer modularity allowed, for the first time, to follow the fate of modules across time-ordered networks. Our results suggest that modules are largely driven by the dominant bird species with a high interaction plasticity to explore available fruit resources in each year. These dynamics suggests that bird-seed dispersal networks may be particularly robust to fluctuations in the availability of interacting partners.

\section{ACKNOWLEDGEMENTS}

We thank the Portuguese ringing authority (CEMPA/ICNF) for providing ringing permits and bird rings. This work was financed by
FCT/MEC through national funds and co-funded by FEDER, within the PT2020 Partnership Agreement and COMPETE 2020 through grants UID/BIA/04004/2019, SFRH/BD/96292/2013 (J.M.C) and SFRH/BD/77746/2011 (L.P.d.S). R.H.H. was partially supported by the Marie Curie Actions FP7-PEOPLE-2012-CIG-321794.

\section{AUTHORS' CONTRIBUTIONS}

R.H.H. and J.A.R. conceived the study; J.M.C., J.A.R., S.T., L.P.d.S., R.S.C. and R.H.H. collected the data; J.M.C. and S.T. performed the analyses; J.M.C. and R.H.H. lead the writing with frequent discussions and input from all authors during the preparation of the manuscript.

\section{DATA AVAILABILITY STATEMENT}

The data and code supporting the results of this study are available from Figshare: https://doi.org/10.6084/m9.figshare.11985912.v1 (Costa et al., 2020).

\section{ORCID}

José M. Costa iD https://orcid.org/0000-0002-4991-7122 Jaime A. Ramos iD https://orcid.org/0000-0002-9533-987X Sérgio Timóteo (iD https://orcid.org/0000-0003-2417-3259 Luís P. da Silva (iD https://orcid.org/0000-0003-2358-1277 Ricardo S. Ceia (iD https://orcid.org/0000-0001-7078-0178 Ruben H. Heleno iD https://orcid.org/0000-0002-4808-4907

\section{REFERENCES}

Aleta, A., \& Moreno, Y. (2019). Multilayer networks in a nutshell. Annual Review of Condensed Matter Physics, 10, 45-62. https://doi. org/10.1146/annurev-conmatphys-031218-013259

Bairlein, F. (2002). How to get fat: Nutritional mechanisms of seasonal fat accumulation in migratory songbirds. Naturwissenschaften, 89, 1-10. https://doi.org/10.1007/s00114-001-0279-6

Bascompte, J., Jordano, P., Melián, C. J., \& Olesen, J. M. (2003). The nested assembly of plant-animal mutualistic networks. Proceedings of the National Academy of Sciences of the United States of America, 100, 9383-9387. https://doi.org/10.1073/pnas.1633576100

Bascompte, J., Jordano, P., \& Olesen, J. M. (2006). Asymmetric coevolutionary networks facilitate biodiversity maintenance. Science, 312, 431-433. https://doi.org/10.1126/science.1123412

Bates, D., Maechler, M., Bolker, B., \& Walker, S. (2015). Fitting linear mixed-effects models using Ime4. Journal of Statistical Software, 67, 1-48. https://doi.org/10.18637/jss.v067.i01

Blondel, V. D., Jean-Loup, G., Renaud, L., \& Etienne, L. (2008). Fast unfolding of communities in large networks. Journal of Statistical Mechanics: Theory and Experiment, 2008, P10008. https://doi.org/ 10.1088/1742-5468/2008/10/P10008

Blonder, B., Wey, T. W., Dornhaus, A., James, R., \& Sih, A. (2012). Temporal dynamics and network analysis. Methods in Ecology and Evolution, 3 , 958-972. https://doi.org/10.1111/j.2041-210X.2012.00236.x

Blüthgen, N., Menzel, F., \& Blüthgen, N. (2006). Measuring specialization in species interaction networks. BCM Ecology, 6, 9. https://doi. org/10.1186/1472-6785-6-9

Brin, S., \& Page, L. (2012). Reprint of: The anatomy of a large-scale hypertextual web search engine. Computer Networks, 56, 3825-3833. https://doi.org/10.1016/j.comnet.2012.10.007

Burkle, L. A., \& Alarcón, R. (2011). The future of plant-pollinator diversity: Understanding interaction networks across time, space, and 
global change. American Journal of Botany, 98, 528-538. https://doi. org/10.3732/ajb.1000391

CaraDonna, P. J., Petry, W. K., Brennan, R. M., Cunningham, J. L., Bronstein, J. L., Waser, N. M., \& Sanders, N. J. (2017). Interaction rewiring and the rapid turnover of plant-pollinator networks. Ecology Letters, 20, 385-394. https://doi.org/10.1111/ele.12740

Carlo, T. A., \& Morales, J. M. (2008). Inequalities in fruit-removal and seed dispersal: Consequences of bird behaviour, neighbourhood density and landscape aggregation. Journal of Ecology, 96, 609-618. https://doi.org/10.1111/j.1365-2745.2008.01379.x

Carnicer, J., Abrams, P. A., \& Jordano, P. (2008). Switching behavior, coexistence and diversification: Comparing empirical community-wide evidence with theoretical predictions. Ecology Letters, 11, 802-808. https://doi.org/10.1111/j.1461-0248.2008.01195.x

Carnicer, J., Jordano, P., \& Melián, C. J. (2009). The temporal dynamics of resource use by frugivorous birds: A network approach. Ecology, 90 1958-1970. https://doi.org/10.1890/07-1939.1

Chacoff, N. P., Resasco, J., \& Vázquez, D. P. (2018). Interaction frequency, network position, and the temporal persistence of interactions in a plant-pollinator network. Ecology, 99, 21-28. https://doi. org/10.1002/ecy.2063

Chao, A. (1987). Estimating the population size for capture-recapture data with unequal catchability. Biometrics, 43, 783-791. https://doi. org $/ 10.2307 / 2531532$

Cirtwill, A. R., Roslin, T., Rasmussen, C., Olesen, J. M., \& Stouffer, D. B. (2018). Between-year changes in community composition shape species' roles in an Arctic plant-pollinator network. Oikos, 127, 11631176. https://doi.org/10.1111/oik.05074

Colwell, R. K. (2013). EstimateS: Statistical estimation of species richness and shared species from samples. Version 9. User's Guide and application published at: http://purl.oclc.org/estimates

Costa, J. M., Ramos, J. A., da Silva, L. P., Timóteo, S., Andrade, P., Araújo, P. M., ... Heleno, R. H. (2018). Rewiring of experimentally disturbed seed dispersal networks might lead to unexpected network configurations. Basic and Applied Ecology, 30, 11-22. https://doi. org/10.1016/j.baae.2018.05.011

Costa, J. M., Ramos, J. A., da Silva, L. P., Timoteo, S., Araújo, P. M., Felgueiras, M. S., ... Heleno, R. H. (2014). Endozoochory largely outweighs epizoochory in migrating passerines. Journal of Avian Biology, 45, 59-64. https://doi.org/10.1111/j.1600-048X.2013.00271.x

Costa, J. M., Ramos, J. A., Timóteo, S., da Silva, L. P., Ceia, R. S., \& Heleno, R. H. (2020). Data from: Species temporal persistence promotes the stability of fruit-frugivore interactions across a 5-year multilayer network. figshare, https://doi.org/10.6084/m9.figshare.11985912.v1

De Domenico, M., Porter, M. A., \& Arenas, A. (2015). MuxViz: A tool for multilayer analysis and visualization of networks. Journal of Complex Networks, 3, 159-176. https://doi.org/10.1093/comnet/cnu038

De Domenico, M., Solé-Ribalta, A., Omodei, E., Gómez, S., \& Arenas, A. (2015). Ranking in interconnected multilayer networks reveals versatile nodes. Nature Communications, 6, 6868. https://doi.org/10.1038/ ncomms7868

Donatti, C. I., Guimarães, P. R., Galetti, M., Pizo, M. A., Marquitti, F. M. D., \& Dirzo, R. (2011). Analysis of a hyper-diverse seed dispersal network: Modularity and underlying mechanisms. Ecology Letters, 14, 773-781. https://doi.org/10.1111/j.1461-0248.2011.01639.x

Donoso, I., García, D., Martínez, D., Tylianakis, J. M., \& Stouffer, D. B. (2017). Complementary effects of species abundances and ecological neighborhood on the occurrence of fruit-frugivore interactions. Frontiers in Ecology and Evolution, 5. https://doi.org/10.3389/fevo.2017.00133

Dormann, C. F., Fründ, J., Blüthgen, N., \& Gruber, B. (2009). Indices, graphs and null models: Analyzing bipartite ecological networks. The Open Ecology Journal, 2, 7-24. https://doi.org/10.2174/1874213000 902010007

Dormann, C. F., Gruber, B., \& Fründ, J. (2008). Introducing the bipartite package: Analysing ecological networks. R News, 8, 8-11.
Dupont, Y. L., Padrón, B., Olesen, J. M., \& Petanidou, T. (2009). Spatiotemporal variation in the structure of pollination networks. Oikos, 118, 1261-1269. https://doi.org/10.1111/j.1600-0706.2009.17594.x

Estes, L., Elsen, P. R., Treuer, T., Ahmed, L., Caylor, K., Chang, J., ... Ellis, E. C. (2018). The spatial and temporal domains of modern ecology. Nature Ecology \& Evolution, 2, 819-826. https://doi.org/10.1038/ s41559-018-0524-4

Galeano, J., Pastor, J. M., \& Iriondo, J. M. (2009). Weighted-Interaction Nestedness Estimator (WINE): A new estimator to calculate over frequency matrices. Environmental Modelling \& Software, 24, 13421346. https://doi.org/10.1016/j.envsoft.2009.05.014

García-Callejas, D., Molowny-Horas, R., \& Araújo, M. B. (2018). Multiple interactions networks: Towards more realistic descriptions of the web of life. Oikos, 127, 5-22. https://doi.org/10.1111/ oik.04428

González-Castro, A., Yang, S., Nogales, M., \& Carlo, T. A. (2012). What determines the temporal changes of species degree and strength in an oceanic island plant-disperser network? PLoS ONE, 7, e41385. https://doi.org/10.1371/journal.pone.0041385

Herrera, C. M. (1984). A study of avian frugivores, bird-dispersed plants, and their interaction in Mediterranean scrublands. Ecological Monographs, 54, 1-23. https://doi.org/10.2307/1942454

Herrera, C. M. (1998). Long-term dynamics of Mediterranean frugivorous birds and fleshy fruits: A 12-years study. Ecological Monographs, 68 , 511-538. https://doi.org/10.2307/2657152

Hutchinson, M. C., Bramon Mora, B., Pilosof, S., Barner, A. K., Kéfi, S., Thébault, E., ... Stouffer, D. B. (2019). Seeing the forest for the trees: Putting multilayernetworks to workfor communityecology. Functional Ecology, 33, 206-217. https://doi.org/10.1111/1365-2435.13237

Jordano, P. (1987). Patterns of mutualistic interactions in pollination and seed dispersal: Connectance, dependence asymmetries, and coevolution. The American Naturalist, 129, 657-677. https://doi. org/10.1086/284665

Jordano, P. (2014). Fruits and frugivory. In R. S. Gallagher (Ed.), Seeds: The ecology of regeneration in plant communities (pp. 18-61). Wallingford, UK: CAB International.

Jutla, I. S., Jeub, L. G. S., \& Mucha, P. J. (2014). A generalized Louvain method for community detection implemented in MATLAB. Retrieved from http://netwiki.amath.unc.edu/GenLouvain (2011-2014).

Koleff, P., Gaston, K. J., \& Lennon, J. J. (2003). Measuring beta diversity for presence-absence data. Journal of Animal Ecology, 72, 367-382. https://doi.org/10.1046/j.1365-2656.2003.00710.x

Newman, M. E. J. (2001). Scientific collaboration networks. II. Shortest paths, weighted networks, and centrality. Physical Review E, 64, 016132. https://doi.org/10.1103/PhysRevE.64.016132

Nicosia, V., \& Latora, V. (2015). Measuring and modeling correlations in multiplex networks. Physical Review E, 92, 032805. https://doi. org/10.1103/PhysRevE.92.032805

Nogales, M., Heleno, R., Rumeu, B., González-Castro, A., Traveset, A., Vargas, P., \& Olesen, J. M. (2015). Seed-dispersal networks on the Canaries and the Galápagos archipelagos: Interaction modules as biogeographical entities. Global Ecology and Biogeography, 25, 912 922. https://doi.org/10.1111/geb.12315

Oksanen, J., Blanchet, F. G., Kindt, R., Legendre, P., Minchin, P. R., O'Hara, R. B., Wagner, H. (2015). Vegan: Community ecology package. R package version, 2.2-1. Nairobi, Kenya: World Agroforestry Centre.

Olesen, J. M., Bascompte, J., Dupont, Y. L., \& Jordano, P. (2007). The modularity of pollination networks. Proceedings of the National Academy of Sciences of the United States of America, 104, 19891-19896. https:// doi.org/10.1073/pnas.0706375104

Olesen, J. M., Dupont, Y. L., O'Gorman, E., Ings, T. C., Layer, K., Melián, C. J., ... Woodward, G. (2010). From Broadstone to Zackenberg: Space, time and hierarchies in ecological networks. Advances in Ecological Research, 42, 1-69. https://doi.org/10.1016/B978-0-12-3813633.00001-0 
Olesen, J. M., Stefanescu, C., \& Traveset, A. (2011). Strong, long-term temporal dynamics of an ecological network. PLOS ONE, 6, e26455. https://doi.org/10.1371/journal.pone.0026455

Opsahl, T. (2009). Structure and evolution of weighted networks (pp. 104122). London, UK: University of London (Queen Mary College).

Opsahl, T. (2013). Triadic closure in two-mode networks: Redefining the global and local clustering coefficients. Social Networks, 35, 159-167. https://doi.org/10.1016/j.socnet.2011.07.001

Patefield, W. M. (1981). Algorithm AS 159: An efficient method of generating random $\mathrm{R} \times \mathrm{C}$ tables with given row and column totals. Journal of the Royal Statistical Society. Series C (Applied Statistics), 30, 91-97.

Payrató-Borràs, C., Hernández, L., \& Moreno, Y. (2019). Breaking the spell of nestedness: The entropic origin of nestedness in mutualistic systems. Physical Review X, 9, 031024. https://doi.org/10.1103/ PhysRevX.9.031024

Petanidou, T., Kallimanis, A. S., Tzanopoulos, J., Sgardelis, S. P., \& Pantis, J. D. (2008). Long-term observation of a pollination network: Fluctuation in species and interactions, relative invariance of network structure and implications for estimates of specialization. Ecology Letters, 11, 564-575. https://doi.org/10.1111/j.1461-0248.2008.01170.x

Pilosof, S., Porter, M. A., Pascual, M., \& Kéfi, S. (2017). The multilayer nature of ecological networks. Nature Ecology \& Evolution, 1, 0101. https://doi.org/10.1038/s41559-017-0101

Plein, M., Längsfeld, L., Neuschulz, E. L., Schultheiß, C., Ingmann, L., Töpfer, T., ... Schleuning, M. (2013). Constant properties of plantfrugivore networks despite fluctuations in fruit and bird communities in space and time. Ecology, 94, 1296-1306. https://doi.org/ 10.1890/12-1213.1

Poisot, T. (2016). betalink: Beta-diversity of species interactions. R package version 2.2.1. Retrieved from https://CRAN.R-project.org/package= betalink

Poisot, T., Canard, E., Mouillot, D., Mouquet, N., \& Gravel, D. (2012). The dissimilarity of species interaction networks. Ecology Letters, 15, 1353-1361. https://doi.org/10.1111/ele.12002

R Core Team. (2017). R: A language and environment for statistical computing. Vienna, Austria: R Foundation for Statistical Computing. Retrieved from http://www.R-project.org/

Ramos-Robles, M., Andresen, E., \& Díaz-Castelazo, C. (2016). Temporal changes in the structure of a plant-frugivore network are influenced by bird migration and fruit availability. PeerJ, 4, e2048. https://doi. org/10.7717/peerj.2048

Rasmussen, C., Dupont, Y. L., Mosbacher, J. B., Trøjelsgaard, K., \& Olesen, J. M. (2013). Strong impact of temporal resolution on the structure of an ecological network. PLoS ONE, 8, e81694. https://doi. org/10.1371/journal.pone.0081694

Rumeu, B., Álvarez-Villanueva, M., Arroyo, J. M., \& González-Varo, J. P. (2019). Interspecific competition for frugivores: Population-level seed dispersal in contrasting fruiting communities. Oecologia, 190, 605-617. https://doi.org/10.1007/s00442-019-04434-9

Schleuning, M., Ingmann, L., Strauß, R., Fritz, S. A., Dalsgaard, B., Matthias Dehling, D., ... Dormann, C. F. (2014). Ecological, historical and evolutionary determinants of modularity in weighted seed-dispersal networks. Ecology Letters, 17, 454-463. https://doi.org/10.1111/ ele.12245

Singmann, H., Bolker, B., Westfall, J., Aust, F., \& Ben-Shachar, M. S. (2020). afex: Analysis of factorial experiments. R package version 0.260 . Retrieved from https://CRAN.R-project.org/package=afex

Thébault, E., \& Fontaine, C. (2010). Stability of ecological communities and the architecture of mutualistic and trophic networks. Science, 329, 853-856. https://doi.org/10.1126/science.1188321

Tilman, D., Isbell, F., \& Cowles, J. M. (2014). Biodiversity and ecosystem functioning. Annual Review of Ecology, Evolution, and Systematics, 45, 471-493. https://doi.org/10.1146/annurev-ecolsys-120213-091917

Timóteo, S., Correia, M., Rodríguez-Echeverría, S., Freitas, H., \& Heleno, R. (2018). Multilayer networks reveal the spatial structure of seed-dispersal interactions across the Great Rift landscapes. Nature Communications, 9, 140. https://doi.org/10.1038/s41467-01702658-y

Traveset, A., Heleno, R. H., \& Nogales, M. (2014). The ecology of seed dispersal. In R. S. Gallagher (Ed.), Seeds: The ecology of regeneration in plant communities (pp. 62-93). Wallingford: CABI.

Trøjelsgaard, K., Jordano, P., Carstensen, D. W., \& Olesen, J. M. (2015). Geographical variation in mutualistic networks: Similarity, turnover and partner fidelity. Proceedings of the Royal Society B: Biological Sciences, 282, 20142925. https://doi.org/10.1098/rspb.2014.2925

Trøjelsgaard, K., \& Olesen, J. M. (2016). Ecological networks in motion: Micro- and macroscopic variability across scales. Functional Ecology, 30, 1926-1935. https://doi.org/10.1111/1365-2435.12710

Wenny, D. G., Şekercioğlu, Ç., Cordeiro, N. J., Rogers, H. S., \& Kelly, D. (2016). Seed dispersal by fruit-eating birds. In Ç. Şekercioğlu, D. G. Wenny, \& C. J. Whelan (Eds.), Why birds matter: Avian ecological function and ecosystem services (pp. 107-145). Chicago, IL: The University of Chicago Press.

Yang, S., Albert, R., \& Carlo, T. A. (2013). Transience and constancy of interactions in a plant-frugivore network. Ecosphere, 4, 147. https:// doi.org/10.1890/ES13-00222.1

\section{SUPPORTING INFORMATION}

Additional supporting information may be found online in the Supporting Information section.

How to cite this article: Costa JM, Ramos JA, Timóteo S, da Silva LP, Ceia RS, Heleno RH. Species temporal persistence promotes the stability of fruit-frugivore interactions across a 5-year multilayer network. J Ecol. 2020;00:1-11. https://doi.org/10.1111/1365-2745.13391 